\title{
Mechanism of Increasing the Permeability of Water-Bearing Coal Rock by Microwave Steam Explosion
}

\author{
Qi Qi $\mathbb{D}^{1,2}$ Weiming Guan $\mathbb{D}^{1,2}$ Xin Li $\mathbb{D}^{1,2}$ Yanyan Ge $\mathbb{C}^{1,2}$ Senlin Nan $\mathbb{D}^{1,2}$ \\ and Huabin Liu $\mathbb{D}^{1,2}$ \\ ${ }^{1}$ College of Geology and Mines Engineering, Xinjiang University, Urumqi 830046, China \\ ${ }^{2}$ Autonomous Region Experimental Teaching Demonstration Center for Geology and Mining Engineering, Xinjiang University, \\ Urumqi 830047, China
}

Correspondence should be addressed to Weiming Guan; gwmxju@xju.edu.cn

Received 2 November 2020; Revised 29 December 2020; Accepted 9 January 2021; Published 30 January 2021

Academic Editor: Bin Gong

Copyright ( 2021 Qi Qi et al. This is an open access article distributed under the Creative Commons Attribution License, which permits unrestricted use, distribution, and reproduction in any medium, provided the original work is properly cited.

\begin{abstract}
Microwave heating of water-bearing coal can promote pore water evaporation. The pores are broken under the action of steam pressure, increasing the permeability of the coal. This study is aimed at investigating the mechanism of permeability improvement of water-bearing coal rock by microwave steam explosion. First, a microwave oven was used to irradiate and heat five groups of coal rock with different water contents; the NMR test was then conducted on the heated sample. Second, the internal vapor pressure and temperature changes during the heating process were obtained through the T-connector for samples with different water contents. Finally, a numerical experiment was used to explore the deformation characteristics of pores under three filling conditions. The experimental results showed that the total porosity increased significantly when the water content of coal increased from $0 \%$ to $8 \%$, while the permeability increased by nearly 4.78 times. The extreme value of gas pressure inside the sample showed an increasing trend. The gas pressure and temperature were in line with the equation of state for an ideal gas during the rising phase. Numerical experiments showed that the pore boundary shrunk inward under vacuum conditions, and compressive stress appeared at the tip. The saturated humid air and liquid water conditions expanded the pore boundaries outward and caused tensile stress at the tip, with the latter being nearly 2.3 times larger than the former, making it more conducive to the development of pores. The findings of this study can be used as a reference value for the expansion of coalbed methane extraction technology.
\end{abstract}

\section{Introduction}

Coalbed methane $(\mathrm{CBM})$ is a source of abundant clean energy $[1,2]$. The recoverable reserves of CBM with a buried depth of less than $2000 \mathrm{~m}$ in China are as high as 10.87 trillion $\mathrm{m}^{3}$. Moreover, CBM is also a hazardous gas in coal mining [3-5] and a pollution source for the greenhouse effect [6]. Therefore, in addition to reducing mine disasters, mining CBM also has huge economic and environmental benefits. However, the low permeability of coal reservoirs is the main factor affecting the efficiency of CBM drainage. Therefore, the enhanced coalbed methane recovery (ECBM) technology is widely proposed [7]. Traditional ECBM technologies mainly include hydraulic fracturing $[8,9]$ and presplit blast- ing [10]. In recent years, many emerging ECBM technologies have been rapidly developed, such as thermal displacement method [11], liquid nitrogen freeze-thaw method [12, 13], pickling method [14], electrochemical method [15], alternative gas injection method [16], sonic excitation method [17], and supercritical carbon dioxide injection $[18,19]$.

Microwave irradiation technology has the characteristics of high thermal efficiency, strong penetrating power, and selective heating. It is used for drying coal [20], pyrolysis [21], improving grindability [22], coking [23], flotation [24], and other aspects. Some studies have reported on the improvement in CBM extraction by microwave irradiation. Most scholars believed that the dielectric constants of different substances in coal reservoirs were quite different. Therefore, the 
efficiency of microwave heating was also quite different, which in turn caused the uneven distribution of the thermal stress field inside the coal reservoir, leading to coal rupture [2528]. Hu et al. [29-31] found that microwave irradiation caused the porosity of coal rocks to increase significantly. On this basis, $\mathrm{Li}$ et al. found that the change in the moisture content had a significant impact on the pore variation behavior [32]. Huang et al. [33] found that water saturation between 25\% and $50 \%$ had the best microwave cracking effect. Huang et al. believed that the increase in water content caused the electric field to be more unevenly distributed and the resulting thermal stress was also more uneven, yielding a better cracking effect [34]. However, the root cause of the increase in coal porosity or permeability under microwave irradiation was not only thermal stress but also the vapor pressure formed by its internal moisture.

This study is aimed at investigating the mechanism of the effect of steam pressure on the pores of coal rock under microwave irradiation. First, a $2 \mathrm{~kW}$ microwave oven was used to irradiate and heat five groups of coal rock samples with different water contents. Then, the variation characteristics of permeability and pore data before and after heating were compared using nuclear magnetic resonance (NMR) test to clarify the overall influence of water content on the coal rock porosity under microwave heating conditions. Then, the internal vapor pressure and temperature variation data of the three groups of coal rock samples with different water content during the heating process were obtained using a T-connector, so as to find out the influence of the water content on the internal vapor pressure and temperature of the pores under microwave heating conditions. Finally, a numerical model of pores under different filling conditions was established to examine the stress and deformation characteristics of pores under the coupling action of steam pressure and thermal stress, so as to reveal the internal mechanism of microwave steam explosion of water-bearing coal rock.

\section{Influence of Water Content on Coal Rock Pores}

2.1. Microwave Heating Experiment Scheme and NMR Test Parameters. Coal blocks were selected from the Fukang mining area in Xinjiang, China, and cylindrical coal samples with a diameter of $\varphi 25 \mathrm{~mm}$ and a height of $50 \mathrm{~mm}$ were produced. The 15 coal rock samples were divided into 5 groups: (1), (2), (3), (4), and (5). The workflow is shown in Figure 1. First, a vacuum drying oven was used to dry the sample for $12 \mathrm{~h}$ at $80^{\circ} \mathrm{C}$. The samples were weighed and recorded after completely drying them. Then, samples (1)-(5) were put in a constanttemperature water bath until saturated. Then, the samples were taken out and placed on an electronic balance to dry naturally until the weights were close to the weights corresponding to the moisture contents of $2 \%, 4 \%, 6 \%$, and $8 \%$, respectively. Finally, samples (1)-(5) were put into the microwave oven, and the microwave power was set to $2000 \mathrm{~W}$ and the heating time to $100 \mathrm{~s}$ for microwave irradiation. After microwave irradiation, the samples were tested by NMR.

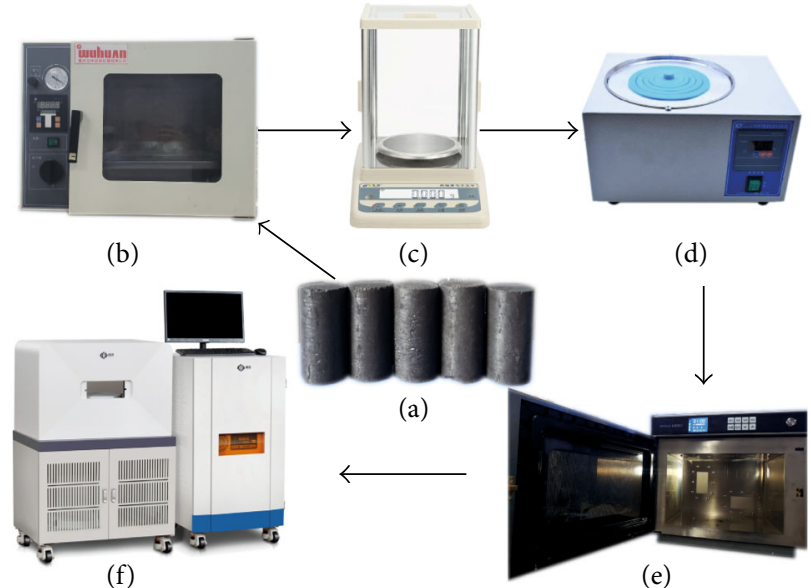

FIGURE 1: Test process of microwave heating of water-bearing coal rock: (a) coal samples, (b) WUHUAN ZK-2020 vacuum drying oven, (c) Di Heng 456A electronic balance, (d) HH-S constanttemperature water bath box, (e) New Sail microwave oven, and (f) MesoMR23-060H NMR testing instrument.

Before the NMR test, the coal rock samples are required to be saturated with water, so as to fill the pores with water. Under the action of a magnetic field, the hydrogen nuclei in water produce NMR. The relaxation time $T_{2}$ is an important signal reflecting the phenomenon of NMR. In the NMR measurement technology, the $T_{2}$ value is directly proportional to a single pore size. Therefore, the relevant data on the size of the internal pore diameter and the proportion distribution of the coal sample can be obtained according to the $T_{2}$ map. The relevant setting parameters of the NMR test in this study are shown in Table 1.

\subsection{Influence of Microwave Irradiation on the Pore Structure} of the Coal Rock. Figures 2(a)-2(e) show the characteristics of the pore radius distribution under different water contents converted from the relaxation time $T_{2}$ spectrum. The horizontal axis represents the pore size, and the vertical axis represents the ratio of pores of a certain diameter to the total pore volume. The area covered by the curve and the horizontal axis is the ratio of the cumulative pore volume to the total pore volume in a certain pore size range. The coal reservoir pores were divided into small pores $(<0.1 \mu \mathrm{m})$, mesopores $(0.1 \mu \mathrm{m}-0.25 \mu \mathrm{m})$, and large pores $(>0.25 \mu \mathrm{m})$. According to this interval, Figures 2(a)-2(e) were integrated to obtain the variation behavior of the proportion of the three pores in the total pore volume under different water contents. As shown in Figure 2(f), the proportion of small pores in the coal rock samples after microwave treatment decreased with the increase in the water content $(62.84 \%, 58.66 \%, 57.22 \%$, $54.47 \%$, and $49.53 \%$ ), suggesting a $21.18 \%$ decrease. The variation in the proportion of mesopores was relatively small (22.38\%, 24.41\%, 25.20\%, 25.40\%, and $24.68 \%)$. The proportion of large pores showed an increasing trend with the increase in the water content $(14.78 \%, 16.93 \%, 17.58 \%$, $20.13 \%$, and $25.79 \%$ ), suggesting a $74.49 \%$ increase.

The wet coal used in the experiment was obtained by the natural drying of saturated coal samples. The longer the 
TABLE 1: NMR test parameter setting table.

\begin{tabular}{lcc}
\hline RF signal frequency main value SF & RF signal frequency offset $O_{1}$ & RF $90^{\circ}$ pulse width $P_{1}$ \\
\hline $23 \mathrm{MHz}$ & $404.68 \mathrm{kHz}$ & $11.52 \mu \mathrm{s}$ \\
RF $180^{\circ}$ pulse width $P_{2}$ & Digital gain $\mathrm{DRG}_{1}$ & Analog gain $\mathrm{RG}_{1}$ \\
$23.52 \mu \mathrm{s}$ & 3 & 20 \\
Accumulated sampling times NS & Echo interval TE & Corresponding pressure of $50 \mathrm{~mm}$ coal rock sample \\
16 & $0.15 \mathrm{~ms}$ & $3.86 \mathrm{MPa}$ \\
Coal core length & Centrifugal speed & Corresponding centrifugal force of $50 \mathrm{~mm}$ coal rock \\
$50 \mathrm{~mm}$ & $8000 /$ min & 560 psi \\
Radio frequency delay RFD & Repeat sampling, interval time TW & Sampling frequency of the signal received by the receiver \\
$0.02 \mathrm{~ms}$ & $2500 \mathrm{~ms}$ & $250 \mathrm{kHz}$ \\
Surface tension at the air/water & Wetting angle at gas-water & Centrifugal force corresponding to pore radius \\
interface & centrifugation & $37 \mathrm{~nm}$ \\
$72 \mathrm{mN} / \mathrm{m}$ & $0^{\circ}$ &
\end{tabular}

drying time, the lower the moisture content. When drying, moisture first escaped from the larger pores and then from the smaller pores in turn. Therefore, small holes were easier to be maintained in the water-filled state compared with other large holes. Also, an increase in the water content caused the number of small holes in the water-filled state to increase, which was similar to the water-bearing state of coal reservoirs under natural conditions. The water in the waterfilled pores was converted into water vapor under the action of a microwave, and more small pores were torn under the action of steam pressure and then converted into mesopores or macropores. Similarly, some mesopores developed into macropores, and the macropores were further enlarged or connected. Therefore, as the moisture content increased, the proportion of small pores continuously reduced, the proportion of mesopores remained basically unchanged, and the proportion of large pores increased greatly.

\subsection{Influence of Microwave Irradiation on Coal Permeability.} Figure 3 shows that the permeability of coal rock samples after microwave fluctuated significantly with the increase in the water content but still followed the overall increasing trend. When the water content of the coal rock samples increased from $0 \%$ to $8 \%$, the permeability after microwave increased from $0.0699 \mathrm{mD}$ to $0.3339 \mathrm{mD}$, an increase of about 4.78 times.

The aforementioned experiments showed that the water content had a great influence on the pore structure of the coal. The two main reasons for the aforementioned experimental results were as follows. On the one hand, the ability to absorb microwaves was stronger because the dielectric loss factor of water was much greater than that of the coal matrix, and it quickly evaporated and formed a steam explosion under high-power microwave irradiation. This highpressure steam promoted the rapid expansion or connection of the internal pore structure of the coal rock sample. On the other hand, the temperature of the coal matrix around the water-bearing pores was higher than that of the other parts. The uneven distribution of thermal stress caused by this was also one reason for the development of pores and cracks.
The permeability of coal rocks greatly improved under the combined effect of the aforementioned reasons, which was more conducive to the extraction of CBM. However, the relationship among vapor pressure, temperature, and water content in the pores was worthy of further discussion.

\section{Internal Temperature and Steam Pressure of Coal Rock}

A T-shaped connection test device was built to test three groups of samples with water contents of $0.1 \%, 2 \%$, and $6 \%$ to investigate the influence of water content on the internal steam pressure and temperature of the pores under microwave heating.

3.1. Test Principle and Experiment Preparation. The test device shown in Figure 4(a) was designed based on the purpose of the experiment. The test device comprised a HT-1890 barometer, a WRNK-131 T-type thermocouple, a high-temperature-resistant nylon pipeline, and a computer. Port 1 of the T-shaped pipeline was connected to the coal rock sample test hole, and port 2 to the differential pressure gauge. The thermocouple was inserted from port 3 through port 1 to the coal rock sample test hole. At the same time, port 3 was made airtight to prevent gas pressure from leaking.

The diameter of the cylindrical sample used in this group of experiments was $\varphi 50 \mathrm{~mm}$, the height was $100 \mathrm{~mm}$, the diameter of the top test hole was $\varphi 8 \mathrm{~mm}$, and the depth was $50 \mathrm{~mm}$. The nine coal rock samples were divided into three groups: (1), (2), and (3), with water contents close to $0.1 \%, 2 \%$, and $6 \%$, respectively, according to the preparation method introduced in Section 2.1 of this study. As shown in Figure 4(b), the samples were put in a nylon tank during the test, and the epoxy sealant was poured into the gap of the sidewall to prevent the sidewall from leaking. Then, a hightemperature-resistant nylon tube with a diameter of $\varphi=6 \mathrm{~mm}$ was inserted, and the connection with epoxy resin was sealed. The microwave oven was set to $2000 \mathrm{~W}$, and the heating time was $100 \mathrm{~s}$. One-to-one corresponding data of 


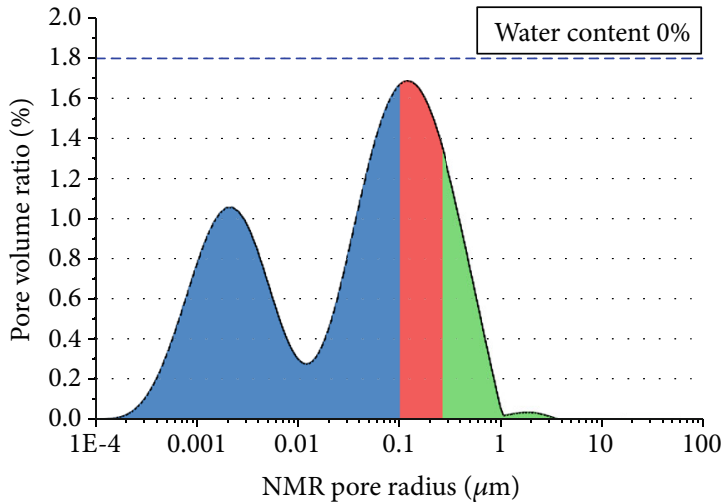

(a)

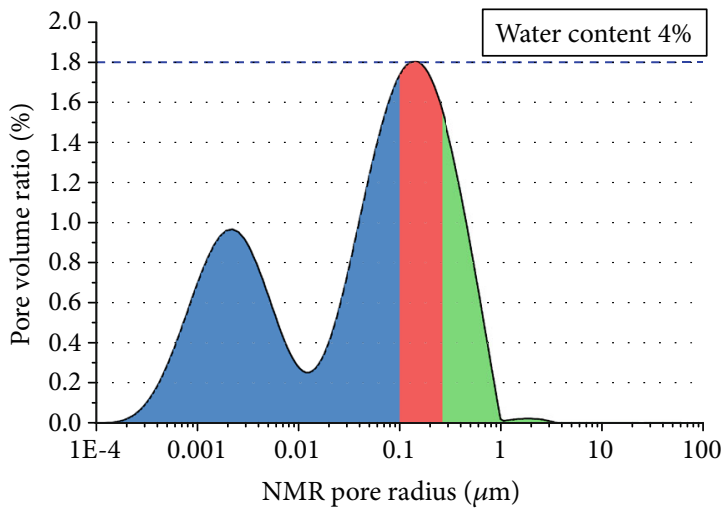

(c)

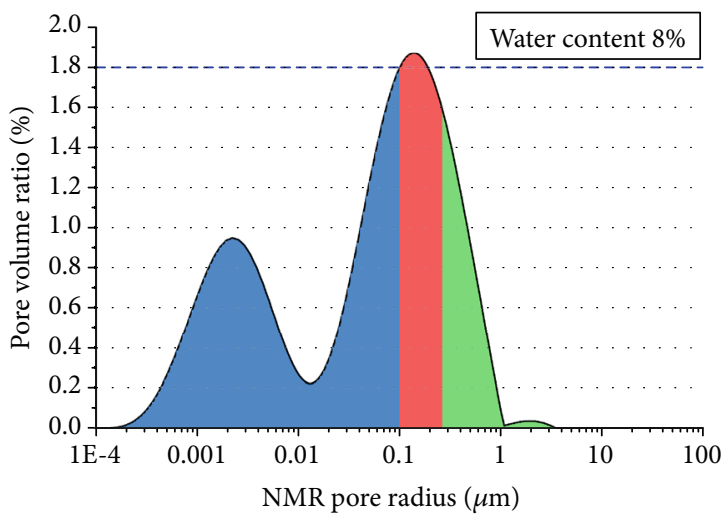

(e)

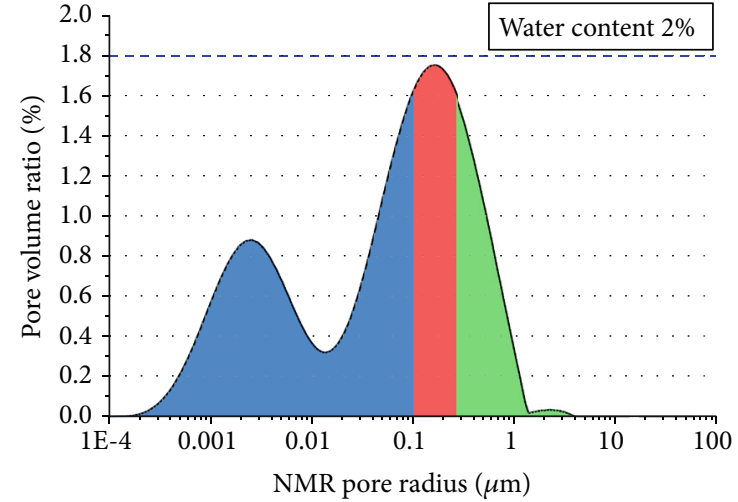

(b)

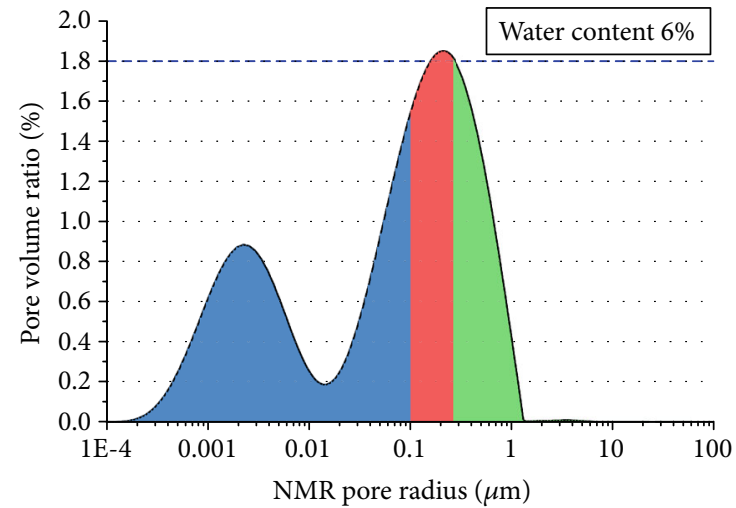

(d)

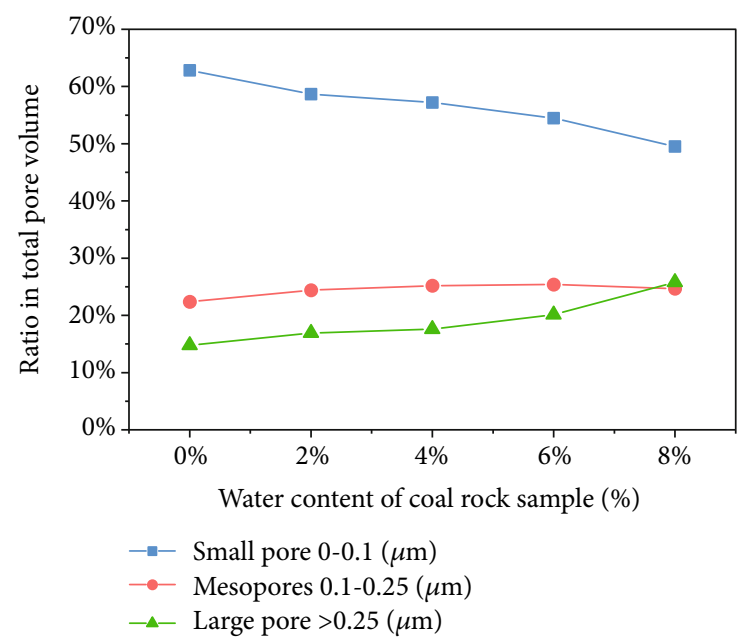

(f)

Figure 2: Pore radius distribution of coal rock samples with different water contents: (a) 0\%, (b) 2\%, (c) 4\%, (d) 6\%, and (e) 8\%. (f) Variations in the proportion of large, medium, and small pore volumes with water contents.

internal temperature and steam pressure are obtained by time consistency of the video.

3.2. Internal Temperature Change of the Coal Rock under Microwave Irradiation. Figure 5 shows that under the same microwave power and irradiation time, the internal temperature of coal rock samples with different water contents showed a trend of rapid increase first and then a slow increase. Also, the heating rate and final temperature increased with an increase in the water content. The temperature of coal samples with water contents of $0.1 \%$, $2.0 \%$, and $6.0 \%$ was $60.0^{\circ} \mathrm{C}, 85.4^{\circ} \mathrm{C}$, and $135.8^{\circ} \mathrm{C}$, respectively, after $100 \mathrm{~s}$.

The dielectric loss factor of water was much greater than that of the coal matrix. The higher the coal rock water content, the greater the overall dielectric loss factor, and the more the electric field energy loss when microwaves penetrated the coal rock mass, which, in turn, generated more 


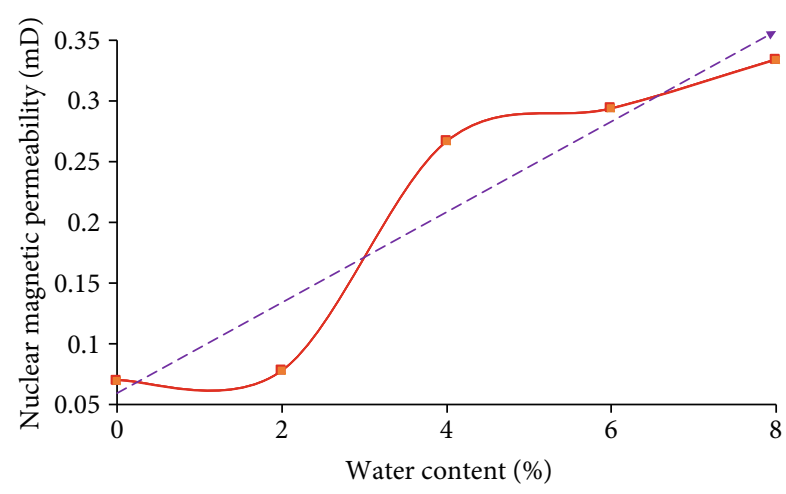

FIgURE 3: Variation in nuclear magnetic permeability of coal rock samples with water content.

heat. Therefore, the higher the water content, the faster the sample heating rate, and the higher the final temperature.

The dielectric loss factor of water vapor was much smaller than that of liquid water. As the experimental temperature increased, the overall dielectric loss factor of the coal rock sample decreased when a large amount of liquid water was converted into steam, and thus, the overall thermal power also significantly reduced. Meanwhile, the heat exchange rate between the surface of the sample and the external environment also increased; therefore, the heating rate of the sample significantly reduced in the latter part of the experiment.

3.3. Changes in the Internal Steam Pressure in Coal Rock under Microwave Irradiation. Figure 6 shows that under the same microwave power and irradiation time, the internal gas pressure of coal samples with different water contents generally showed a trend of first increase and then decrease. The extreme values of gas pressure increased with the increase in the water content $(0.1 \%, 2.0 \%$, and $6.0 \%)(128.8 \mathrm{kPa}, 165.3 \mathrm{kPa}$, and $319.6 \mathrm{kPa}$ ). The arrival time of the extreme value was earlier (50s, $82 \mathrm{~s}$, and $90 \mathrm{~s})$ as the water content increased.

Gas pressure was closely related to temperature. The higher the moisture content of the coal sample, the faster the rise in temperature, resulting in a faster increase in the gas pressure rise and a higher peak value. As the internal pressure continued to rise, the pressure difference between the inside and outside of the coal sample became larger, destroying the original mechanical balance of the hole/fracture. As a result, the pores and cracks inside the coal sample continued to expand and gradually connected with the outside world. Then, the pressure gradually decreased after reaching the peak value.

3.4. Relationship between Coal Temperature and Steam Pressure under Microwave Irradiation. During the microwave heating process, the evaporation process in the entire coal rock sample was considered to be carried out in a closed environment with a fixed volume when the pores of the coal rock were not ruptured on a large scale. Therefore, the equation of state for an ideal gas was used to calculate the gas pressure.

$$
P=C_{e} R T,
$$

where $P$ is the pressure of the ideal gas, in Pa; $T$ is the thermodynamic temperature of the ideal gas, in $\mathrm{K} ; R$ is the ideal gas constant, $8.314 \mathrm{~J} /(\mathrm{mol} \cdot \mathrm{K})$; and $C_{e}$ is the molar concentration of the ideal gas, in $\mathrm{mol} / \mathrm{m}^{3}$. The liquid water in the pores continuously provided water vapor as the temperature increased, due to the small volume of coal pores. Therefore, it was considered that the water vapor in the pores of coal was always saturated. The molar concentration of saturated water vapor at each temperature was obtained according to [35]

$$
C_{V}=\frac{610.7 \times 10^{7.5 \times((T-273.15) /(T-35.85))}}{8.3145 \times T} .
$$

At the beginning of this experiment, the pores were filled with saturated humid air composed of dry air and water vapor. As the temperature increased, liquid water evaporated into water vapor, and the molar concentration of water vapor in the pores continued to increase, while the molar concentration of dry air did not change. Therefore, the molar concentration of humid air in the pores should be the sum of the initial molar concentration of dry air and the current molar concentration of water vapor. Under initial conditions $\left(25^{\circ} \mathrm{C}, 1 \mathrm{~atm}\right)$, the molar concentration of dry air was $C_{A}=41.58 \mathrm{~mol} / \mathrm{m}^{3}$ and the molar concentration of water vapor $C_{V}$ was calculated according to equation (2). The humid gas pressure under different temperature conditions was calculated by substituting $C_{e}=C_{A}+C_{V}$ into equation (1). Figure 7 shows the comparison between the calculated pressure and the measured pressure in the $50 \mathrm{~s}$ period before heating under the three moisture contents. The average errors were $1.77 \%, 2.12 \%$, and $7.92 \%$, respectively. This finding indicated that the internal temperature rise and gas pressure increase of the microwave-heated water-bearing coal generally agreed with the equation of state of an ideal gas. Datta [36] also found a similar conclusion in the study of simultaneous heat and mass transfer in porous media.

Physical experiments verified that the gas pressure inside the coal rock under microwave irradiation could promote the further development of pores, and the pressure and temperature of the wet air in the coal rock pores conformed to the equation of state of the ideal gas. However, how coal rock pores deformed under the coupling effect of steam pressure and thermal stress still remained unanswered. Therefore, whether the different forms of moisture inside the pores affected the deformation needed further study.

\section{Numerical Analysis of Pore Deformation of Coal Rock}

4.1. Model Establishment and Experimental Setup. The force and deformation of the internal pores of coal rock could not be easily observed through physical experiments. Therefore, the COMSOL multiphysics numerical software was used to establish a single-hole numerical model under different pore-filling conditions (vacuum, saturated humid air, and liquid water). The model established a two-dimensional rectangular model based on the largest cross-section of the coal rock specimen (height $100 \mathrm{~mm}$ and width $50 \mathrm{~mm}$ ), with an 


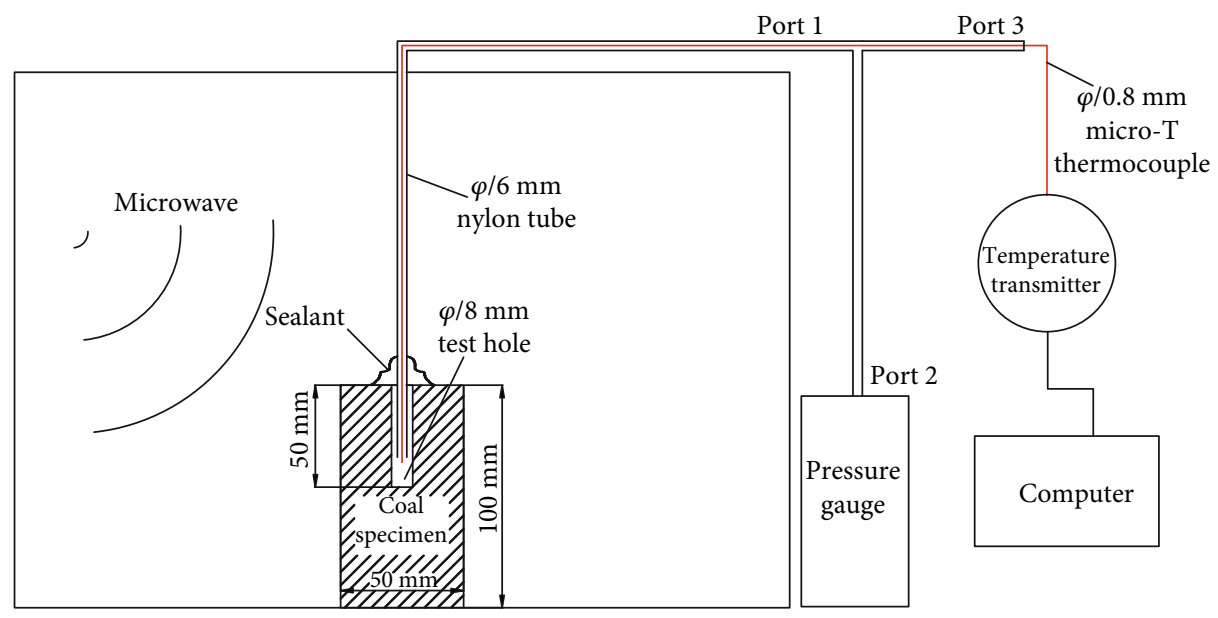

(a)

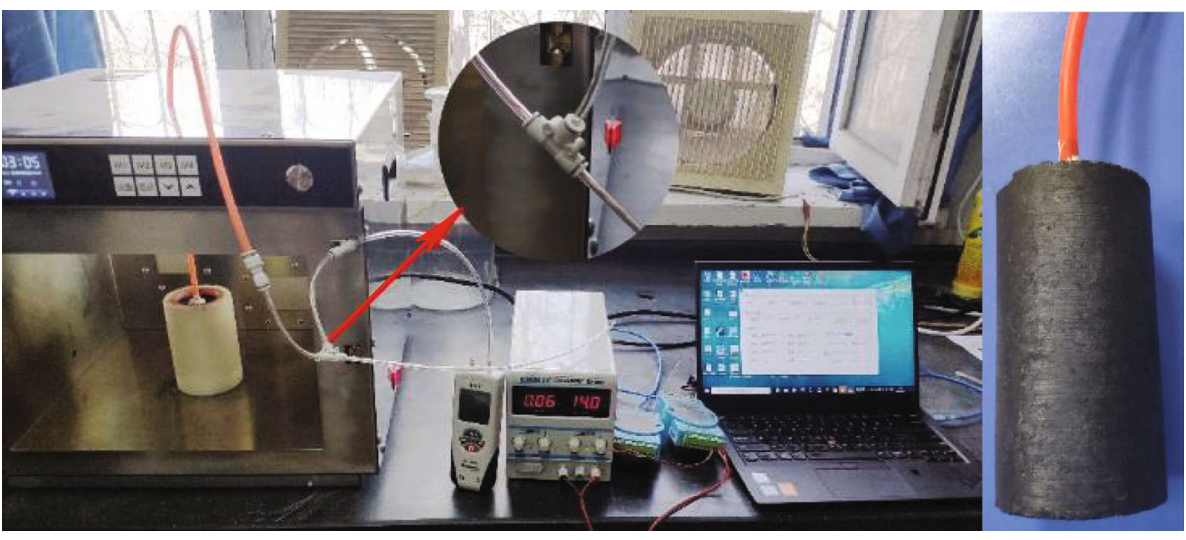

(b)

FIGURE 4: Gas pressure and temperature test system for microwave heating of water-bearing coal rock: (a) principle of the test device and (b) test device and coal rock sample.

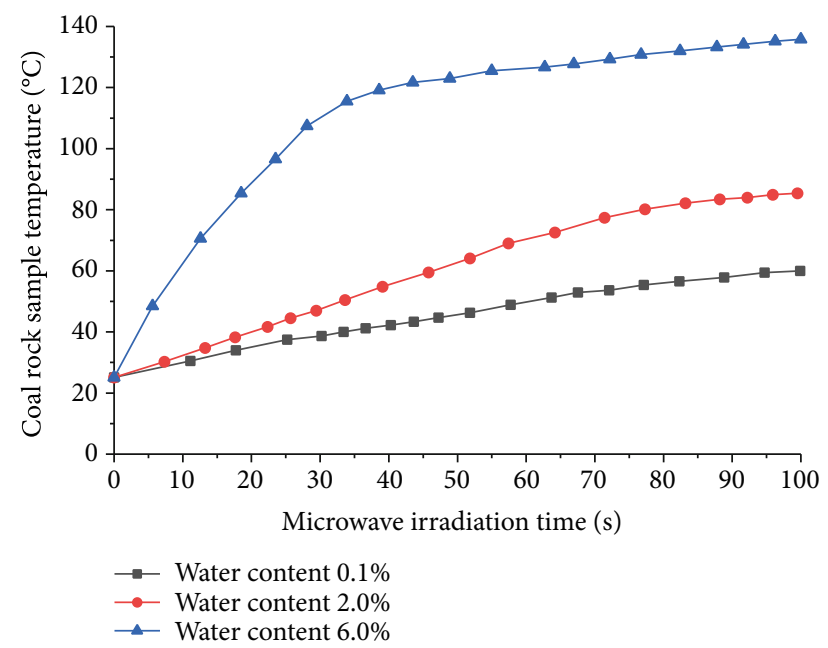

FIGURE 5: Changes in internal temperature with time of coal rock samples with different water contents.

elliptical pore area in the middle. Its major semiaxis was $25 \mathrm{~mm}$, and the minor semiaxis was $1 \mathrm{~mm}$.

The gas pressure in the pore area was calculated according to the equation of state for an ideal gas equation (1) and acted on the pore wall in the form of pressure load. The bottom boundary of the rectangular model could only deform upward by limiting the displacement, and the other boundaries were free.

The electric heat force multifield was solved by one-way coupling. First, the electric field was solved in the frequency domain. Then, the transient solution of the temperature field was performed according to the intensity distribution of the electric field. Finally, the thermal stress and ideal gas pressure were calculated according to the temperature field to obtain the distribution of the stress field in the specimen and the characteristics of pore deformation.

4.2. Analysis of Numerical Experiment Results. The thermodynamic, electrical, and mechanical parameters of the coal matrix were set to the same values to compare the stress and deformation characteristics of the pores under different filling conditions. Also, the numerical samples were heated for the same time under the same microwave conditions to obtain the same temperature field distribution characteristics. The different pore deformations generated under this condition should only be related to the internal gas pressure. As shown in Figures 8(a)-8(c), the deformation of the vacuum pores was inward shrinkage and the other two 


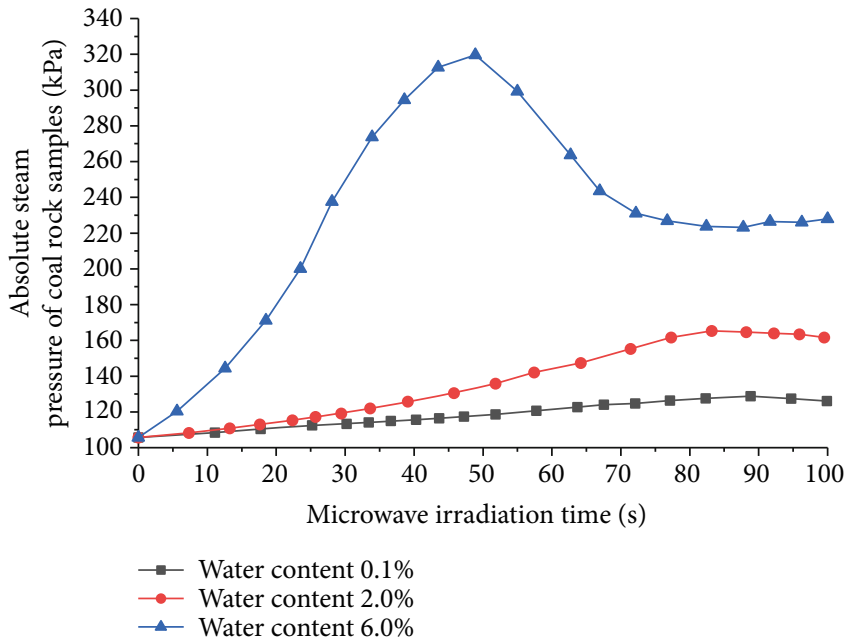

FigurE 6: Changes in the internal absolute gas pressure with time of coal rock samples with different water contents.

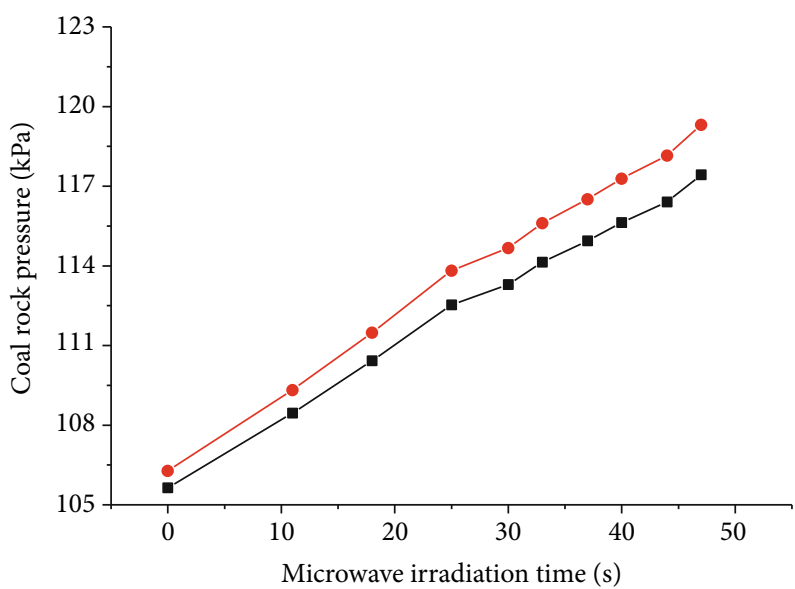

(a)

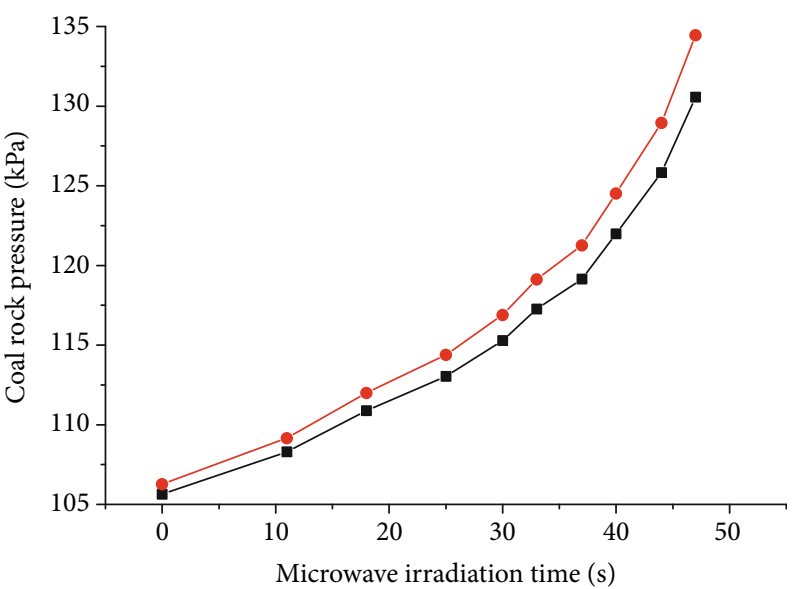

(b)

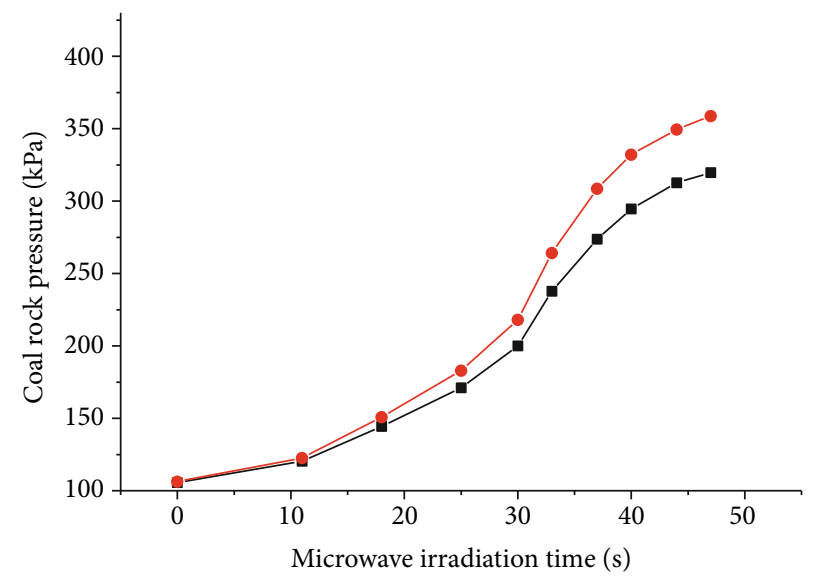

- Measured coal rock pressure with water content of $0.1 \%$

$\rightarrow$ - Theoretically calculated coal rock pressure with water content of $0.1 \%$

(c)

FiguRE 7: Comparison of calculated and tested vapor pressure values under different water contents: (a) $0.1 \%$, (b) $2 \%$, and (c) $6 \%$. 


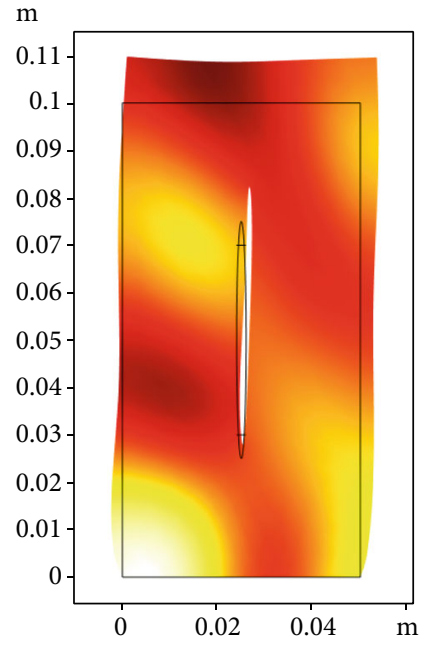

(a)

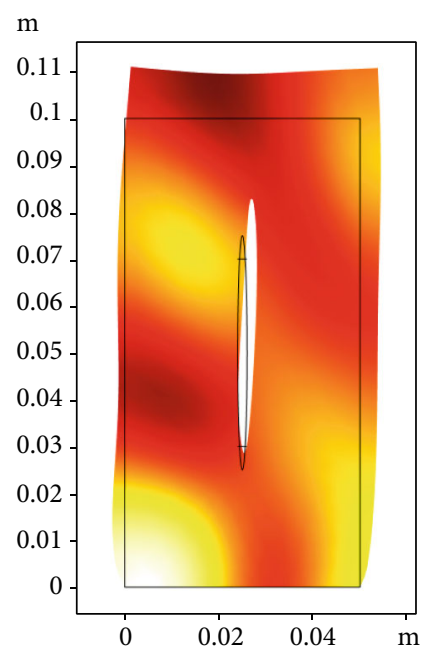

(c)

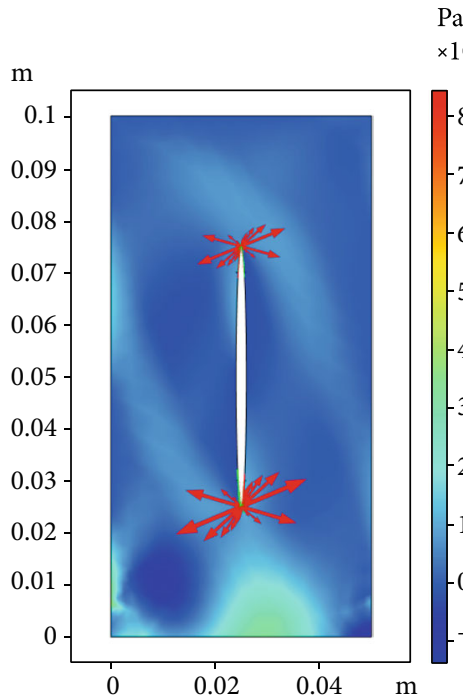

(e) $\operatorname{deg} \mathrm{C} m$

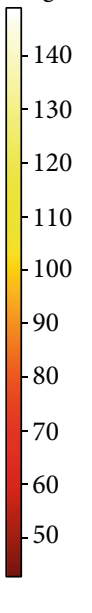

140

120

$\begin{array}{ll}110 & 0.07\end{array}$

$00 \quad 0.06-$

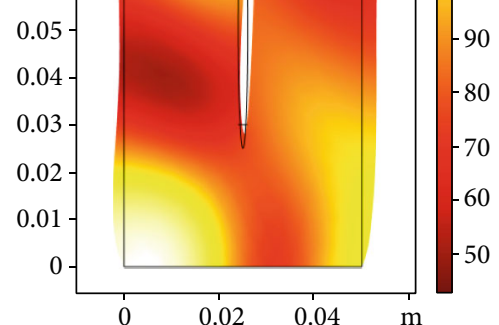

(b)

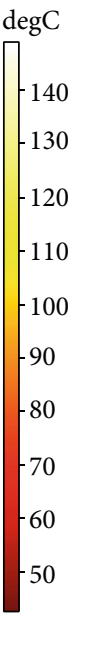

egC $\mathrm{m}$

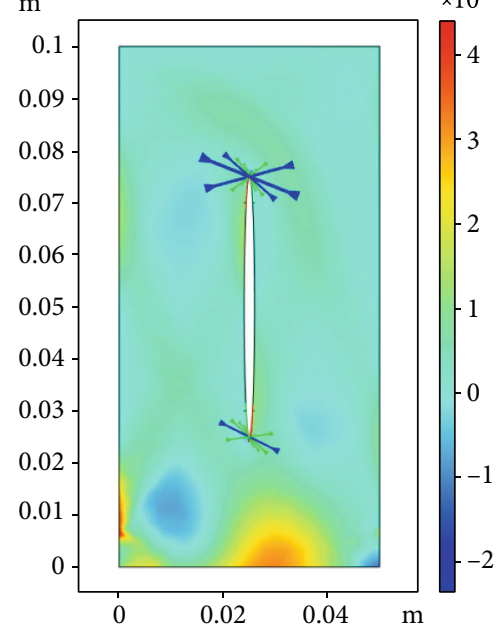

(d)

$\mathrm{Pa}$

$\times 10^{5}$

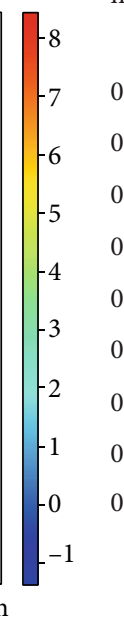

$0.1-$

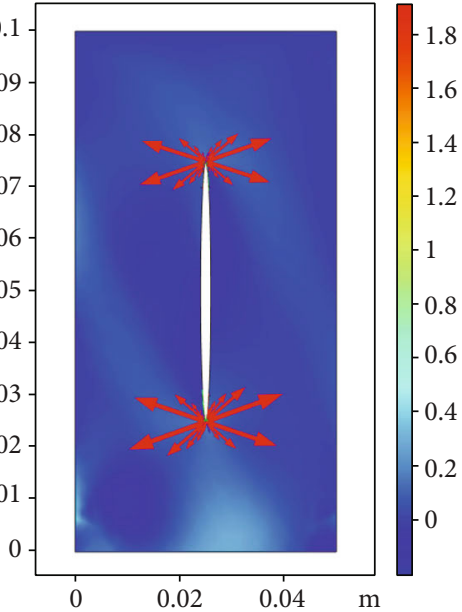

(f)

$\operatorname{deg} \mathrm{C}$

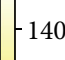

$-130$

120

110

100

80

70

60 政

4

3

$-1$

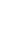




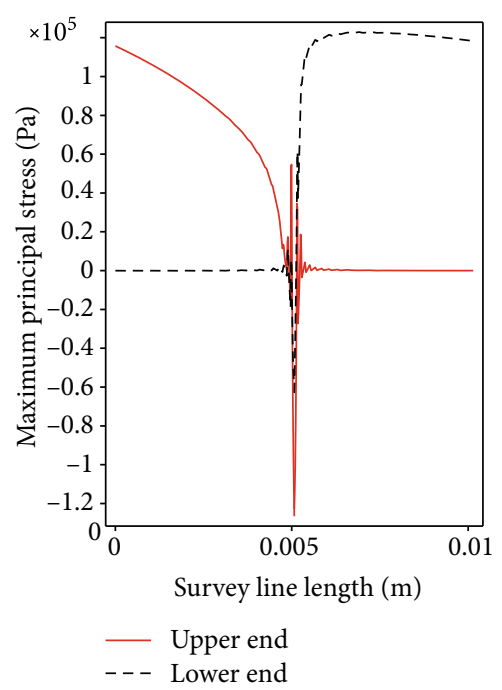

(g)

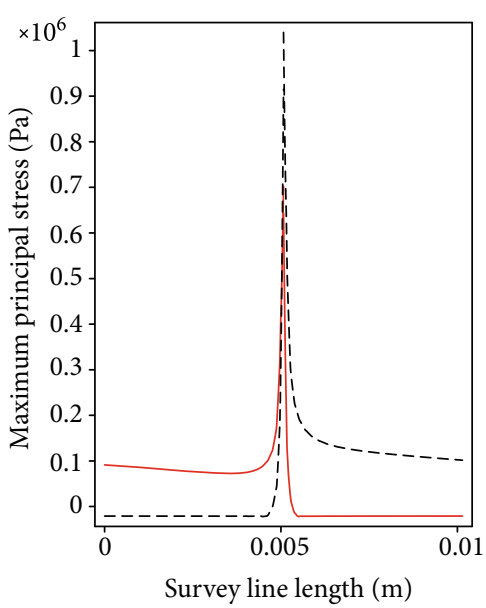

(h)

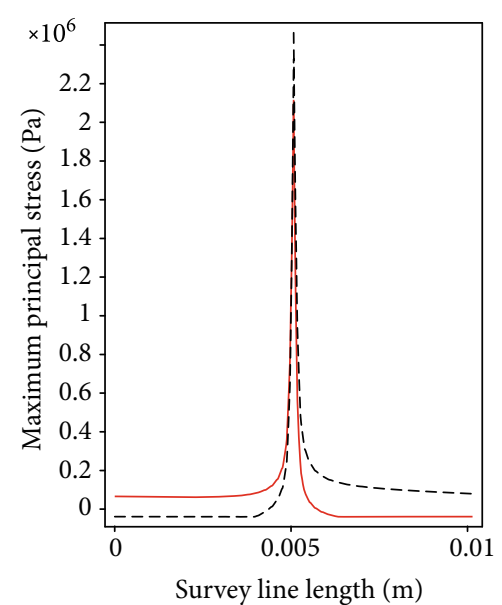

(i)

Figure 8: $(\mathrm{a}-\mathrm{c})$ Temperature and pore deformation characteristics of vacuum pores, saturated humid air pores, and liquid-containing pores. (d-f) Maximum principal stress distribution and stress direction characteristics. (g-i) Distribution of the maximum principal stress at the upper- and lower-tip contours.

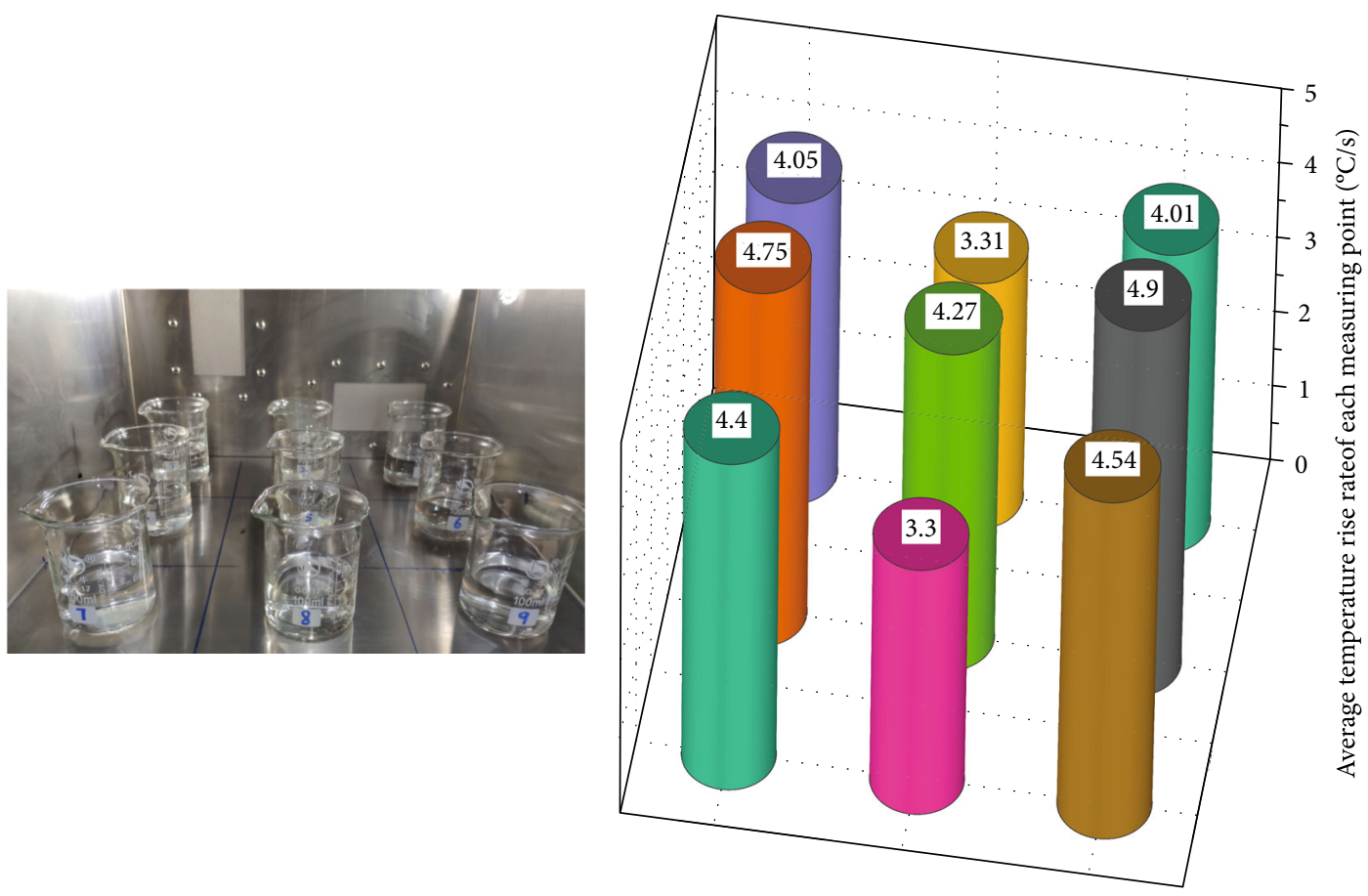

(a)

(b)

Figure 9: (a) Microwave electric field intensity test by the water-loading method. (b) Distribution of test results of the heating rate in the microwave oven.

conditions were outward expansion, with the outward expansion of the pores containing liquid water being more significant. Figures $8(\mathrm{~d})-8(\mathrm{f})$ show the distribution of the maximum principal stress under the three conditions. The force on the tip of the vacuum pore was found to be mainly compressive stress, while it was tensile stress under the other two conditions. Figures $8(\mathrm{~g})-8(\mathrm{i})$ shows the maximum prin- cipal stress distribution on the outer contours of the upper and lower tips of the pores under the three filling conditions. The maximum principal stress at the tip under vacuum conditions was $-0.12 \mathrm{MPa}$, while it was $1.1 \mathrm{MPa}$ and $2.5 \mathrm{MPa}$, respectively, under the other two filling conditions. The numerical results of this stress might vary with the pore structure or other mechanical boundary conditions. However, the 
TABLE 2: Calculation results of the electric field intensity of the water cup load experiment.

\begin{tabular}{lccccccccc}
\hline \multicolumn{1}{l}{$\boldsymbol{L} T / t\left({ }^{\circ} \mathrm{C} / \mathrm{s}\right)$} & \multicolumn{3}{c}{$P_{w}\left(\mathrm{~kW} / \mathrm{m}^{3}\right)$} & & $E(\mathrm{~V} / \mathrm{m})$ & Average electric field strength $(\mathrm{V} / \mathrm{m})$ \\
\hline 4.05 & 3.31 & 4.01 & 17,010 & 13,902 & 16,842 & 3950.60 & 3571.49 & 3931.04 & 4000.01 \\
4.75 & 4.27 & 4.9 & 19,950 & 17,934 & 20,580 & 4278.40 & 4056.48 & 4345.43 & \\
4.4 & 3.3 & 4.54 & 18,480 & 13,860 & 19,068 & 4117.76 & 3566.09 & 4182.76 & \\
\hline
\end{tabular}

increase in the internal steam pressure was more beneficial to the development of pores or the flow of CBM to a certain extent.

Heat was transferred from the inside to the outside of the coal rock during microwave heating. When the pores were a vacuum, the thermal expansion of the coal matrix near the pores was more likely to develop in the inward direction of the pores, thus forming a shrinking deformation feature. When the pores contained saturated humid air, the gas pressure acting on the pore walls increased with the increase in temperature. When the pores contained liquid water, more water vapor was generated and acted on the pore walls as the temperature in the pores continued to increase. Therefore, a greater vapor pressure was formed in the pores, and thus, the tensile stress on the tip of the void further increased. The tensile strength of the general rock was much smaller than the compressive strength. Therefore, using this method was more conducive to the development of the pore structure of coal reservoirs.

\section{Calculation Method for a Temperature Rise of Coal Rock under Microwave}

5.1. Calculation of the Heating Rate of Microwave Heating. The aforementioned experiments proved that the water vapor formed by the moisture inside the coal rock under microwave irradiation could promote the deformation and development of coal rock pores. The transformation of electromagnetic energy into thermal energy was the root cause of the aforementioned changes. According to previous studies, the electromagnetic power loss could be calculated according to [37]

$$
P_{w}=2 \pi f \varepsilon_{r}^{\prime \prime} \varepsilon_{0}|E|^{2}
$$

where $P_{w}$ is the electromagnetic loss thermal power density, in $\mathrm{W} / \mathrm{m}^{3}$, and $f$ is the electric field frequency, in Hz. $\varepsilon_{r}^{\prime \prime}$ is the imaginary part of the relative complex permittivity of the heated object, which characterizes the material's ability to absorb and lose electromagnetic field energy. $\varepsilon_{0}$ is the dielectric constant in vacuum $8.85 e^{-12} \mathrm{~F} / \mathrm{m}$, and $|E|$ is the modulus of electric field strength, in $\mathrm{V} / \mathrm{m}$.

Meanwhile, the heating power of the heated object could also be calculated according to the thermodynamic equation (4). According to the law of conservation of energy, equations (3) and (4) should be equal. Therefore, equations (3) and (4) were combined to obtain equation (5) for calculating the heating rate.

$$
\begin{aligned}
P_{w} & =\rho C_{p} \frac{\Delta T}{t}, \\
\frac{\Delta T}{t} & =\frac{2 \pi f \varepsilon_{r}^{\prime \prime} \varepsilon_{0}|E|^{2}}{\rho C_{p}} .
\end{aligned}
$$

TABle 3: Thermodynamic and electrical parameters of mixed substances [39-44].

\begin{tabular}{lccc}
\hline Substance & $\rho_{i}\left(\mathrm{~kg} / \mathrm{m}^{3}\right)$ & $C_{p i}\left[\mathrm{~J} /\left(\mathrm{kg} .{ }^{\circ} \mathrm{C}\right)\right]$ & $\varepsilon_{r i}^{\prime \prime}$ \\
\hline Water & 1000 & 4200 & 8 \\
Air & 1.205 & 1200 & 0 \\
Coal & 1200 & 1200 & 2 \\
\hline
\end{tabular}

Since coal rock is a kind of mixture, its dielectric loss factor could be estimated according to the dielectric loss factor of each substance and their volume ratio [38], such that $\varepsilon_{r}^{\prime \prime}=\sum_{i=1}^{n} V_{i} \varepsilon_{r i}^{\prime \prime}$, where $V_{i}$ is the volume ratio of a certain substance. Similarly, the density of coal rock mass was calculated as $\rho=\sum_{i=1}^{n} V_{i} \rho_{i}$, in $\mathrm{kg} / \mathrm{m}^{3}$. The specific heat capacity of coal rock mass was calculated as $C_{p}=\left(\sum_{i=1}^{n} V_{i} \rho_{i} C_{p i}\right) / \rho$, in $\mathrm{J} /\left(\mathrm{kg} .{ }^{\circ} \mathrm{C}\right)$, where $t$ is the heating time, in $s$. From equation (5), the average temperature of the water-bearing coal rock mass after heating for a certain period of time could be obtained.

5.2. Verification of the Calculation Method of Heating Rate. In this experiment, an indirect estimation was made using the water cup load method because the electric field strength in the microwave oven could not be tested with an instrument. Since the electric field intensity distribution in the microwave oven was not uniform, it was equally divided into nine areas. Three water cup load experiments were carried out for each position to obtain the electric field intensity at each position. As shown in Figure 9(a), first, a beaker containing $50 \mathrm{~mL}$ of water was placed at position 1 for heating. The heating power of the microwave oven was set to $2000 \mathrm{~W}$, and the heating time was set to $10 \mathrm{~s}$. After heating, the temperature rise $\Delta T$ was calculated (the initial temperature was $20^{\circ} \mathrm{C}$ ) and recorded. Then, the aforementioned experiment was carried out at the remaining eight positions in turns. Substituting the average value of the three temperature rises $\Delta T$ at each location into equation (6), the electric field strength $E$ at each location could be calculated:

$$
|E|=\left(\frac{\rho C_{p} \Delta T}{2 \pi f \varepsilon_{r}^{\prime \prime} \varepsilon_{0} t}\right)^{1 / 2}
$$

As the specific heat capacity, density, and dielectric constant of water were tested in other related studies $[39,40]$, their values were taken as $C_{p}=4200 \mathrm{~J} /\left(\mathrm{kg} \cdot{ }^{\circ} \mathrm{C}\right), \rho=1000$ $\mathrm{kg} / \mathrm{m}^{3}$, and $\varepsilon_{r}^{\prime \prime}=8$. Therefore, only the rate of change in temperature increase $\Delta T / t$ in equation (4) was required to obtain the electric field intensity. Figure 9(b) shows the heating rate distribution in $10 \mathrm{~s}$ at each position. Based on this, the calculated electric field intensity is shown in Table 2. 
TABLE 4: Temperature rise rate of coal rock samples with different water contents under microwave irradiation.

\begin{tabular}{|c|c|c|c|c|c|c|c|c|c|c|}
\hline \multirow{2}{*}{$\begin{array}{l}\text { Water } \\
\text { content (\%) }\end{array}$} & \multicolumn{3}{|c|}{ Volume ratio (\%) } & \multicolumn{3}{|c|}{$\begin{array}{l}\text { Effective parameters of coal rock } \\
\text { mass }\end{array}$} & \multirow{2}{*}{$\begin{array}{l}\Delta T / t\left({ }^{\circ} \mathrm{C} / \mathrm{s}\right), \text { initial } \\
\text { temperature } 25^{\circ} \mathrm{C}\end{array}$} & \multirow{2}{*}{$\begin{array}{c}\text { Predicted } \\
\text { temperature }\left({ }^{\circ} \mathrm{C}\right)\end{array}$} & \multirow{2}{*}{$\begin{array}{c}\text { Measured } \\
\text { temperature }\left({ }^{\circ} \mathrm{C}\right)\end{array}$} & \multirow{2}{*}{$\begin{array}{c}\text { Error } \\
(\%)\end{array}$} \\
\hline & Water & Air & Coal & $\begin{array}{c}P \\
\left(\mathrm{~kg} / \mathrm{m}^{3}\right)\end{array}$ & $\begin{array}{c}C_{p} \\
\left(\mathrm{~J} /\left(\mathrm{kg} \cdot{ }^{\circ} \mathrm{C}\right)\right)\end{array}$ & $\begin{array}{c}\varepsilon_{r}^{\prime \prime} \\
\left(\mathrm{C}^{2} /\left(\mathrm{N} \cdot \mathrm{M}^{2}\right)\right]\end{array}$ & & & & \\
\hline 0.1 & 0.09 & 7.83 & 92.08 & 1105.95 & 1202.44 & 0.19 & 0.30 & 56.37 & 60.00 & 6.06 \\
\hline 2 & 1.84 & 6.08 & 92.08 & 1123.43 & 1249.14 & 0.33 & 0.49 & 76.47 & 85.40 & 10.46 \\
\hline 6 & 5.52 & 2.40 & 92.08 & 1160.19 & 1342.74 & 0.63 & 0.83 & 112.56 & 135.80 & 17.11 \\
\hline
\end{tabular}

Coal rock mass is a mixture of moisture, air, and coal matrix. When the moisture content changes, the volume ratio of each substance also changes. The imaginary parts of the density, specific heat capacity, and relative complex permittivity of the three substances are shown in Table 3 . Substituting them and the average electric field intensity in the microwave oven obtained by the water load experiment $E=4000.01 \mathrm{~V} / \mathrm{m}$ into equation (5), the average temperature of the coal rock mass with different water contents after $100 \mathrm{~s}$ could be obtained. Table 4 shows that the average error between the calculated temperature and the measured temperature was $11.21 \%$. This was because the temperature measurement position was in the central hole of the coal rock. When the temperature was high, the sensor felt the steam temperature, so the measured temperature was higher. However, equation (5) could accurately predict the average temperature of coal rock masses with different water contents irradiated by microwave. At the same time, since the vapor pressure and temperature generally accorded with the equation of state for an ideal gas during the temperature rising stage, the vapor pressure can be calculated after the temperature rise rate is obtained.

\section{Conclusions}

This study considered the promotion of the deformation and development of coal rock pores by high-pressure water vapor formed by the evaporation of water inside the coal rock under microwave irradiation. Related experiments were carried out for analysis, and the following conclusions were obtained:

(1) The increase in water content promoted more water filling in the tiny pores. The pressure formed by the evaporation of water under microwave irradiation promoted further development of more pores into larger pores. In this experiment, the total porosity increased significantly with the increase in the water content. The largest increase in large pores was about $74.49 \%$, and the permeability increased by nearly 4.78 times

(2) The water content affected the overall dielectric loss factor of coal rock and changed the thermal sensitivity of coal rock to microwaves. Therefore, the higher the coal rock water content, the faster the temperature and pressure increase inside the pores. In addition, the vapor pressure and temperature generally accorded with the equation of state for an ideal gas during the temperature rising stage
(3) The steam pressure inside the pores of coal was related to its filling state. The pores containing liquid water provided more vapor material sources during the heating process and thus could generate greater gas pressure than dry pores containing only saturated humid air. Under the action of this pressure, greater tensile stress was easily generated at the tip of the pore, making the pore more easily torn through

(4) The heat loss of microwave electromagnetic energy in coal rock was the main energy source for pore deformation due to water evaporation. However, the coal rock mass is a mixture of substances, and its electrical and thermodynamic properties could be calculated based on the volume ratio of the main components. The heating rate could be estimated according to $\Delta T / t=\left(2 \pi f \varepsilon_{r}^{\prime \prime} \varepsilon_{0}|E|^{2}\right) /\left(\rho C_{p}\right)$

\section{Data Availability}

The data that support the findings of this study are available on request from the corresponding author. The data are not publicly available.

\section{Conflicts of Interest}

The authors declare that there are no conflicts of interest regarding the publication of this paper.

\section{Acknowledgments}

The research is financially supported by the National Natural Science Foundation of China (No. 51764050), PhD Research Startup Fund of Xinjiang University (No. BS1902013), and National Natural Science Foundation of China (No. 42002188 and No. 42062012).

\section{References}

[1] U. S. EIA, Natural gas gross withdrawals and production, vol. 18, Gross Withdrawals, Annual, 2013.

[2] F. Zhou, T. Xia, X. Wang, Y. Sun, J. Liu, and J. Liu, "Recent developments in coal mine methane extraction and utilization in China: a review," Journal of Natural Gas Science and Engineering, vol. 31, pp. 437-458, 2016.

[3] H. Li, B. Lin, W. Yang, Y. Gao, and T. Liu, "Effects of an underlying drainage gallery on coal bed methane capture effectiveness and the mechanical behavior of a gate road," Journal of 
Natural Gas Science and Engineering, vol. 27, pp. 616-631, 2015.

[4] N. SKOCZYLAS, "Laboratory study of the phenomenon of methane and coal outburst," International Journal of Rock Mechanics and Mining Sciences, vol. 55, pp. 102-107, 2012.

[5] M. B. Wold, L. D. Connell, and S. K. Choi, "The role of spatial variability in coal seam parameters on gas outburst behaviour during coal mining," International Journal of Coal Geology, vol. 75, no. 1, pp. 1-14, 2008.

[6] K. WARMUZINSKI, "Harnessing methane emissions from coal mining," Process Safety and Environmental Protection, vol. 86, no. 5, pp. 315-320, 2008.

[7] A. Busch and Y. Gensterblum, "CBM and CO2-ECBM related sorption processes in coal: a review," International Journal of Coal Geology, vol. 87, no. 2, pp. 49-71, 2011.

[8] J. Zhang, "Numerical simulation of hydraulic fracturing coalbed methane reservoir," Fuel, vol. 136, pp. 57-61, 2014.

[9] Q. Li, B. Lin, and C. Zhai, "The effect of pulse frequency on the fracture extension during hydraulic fracturing," Journal of Natural Gas Science and Engineering, vol. 21, pp. 296-303, 2014.

[10] W. Zhu, D. Gai, C. Wei, and S. Li, "High-pressure air blasting experiments on concrete and implications for enhanced coal gas drainage," Journal of Natural Gas Science and Engineering, vol. 36, pp. 1253-1263, 2016.

[11] Y. Cai, D. Liu, Y. Yao, Z. Li, and Z. Pan, "Partial coal pyrolysis and its implication to enhance coalbed methane recovery, part I: an experimental investigation," Fuel, vol. 132, pp. 12-19, 2014.

[12] H. Yan, L. Tian, R. Feng, J. Z. Chen, B. Zhang, and B. Zhang, "Fracture evolution in coalbed methane reservoirs subjected to liquid nitrogen thermal shocking," Journal of Central South University, vol. 27, no. 6, pp. 1846-1860, 2020.

[13] C. Cai, G. Li, Z. Huang, S. Tian, J. Wei, and J. Wei, "Experimental study of the effect of liquid nitrogen cooling on rock pore structure," Journal of Natural Gas Science and Engineering, vol. 21, pp. 507-517, 2014.

[14] L. G. Turner and K. M. Steel, "A study into the effect of cleat demineralisation by hydrochloric acid on the permeability of coal," Journal of Natural Gas Science and Engineering, vol. 36, pp. 931-942, 2016.

[15] J. Guo, T. Kang, J. Kang, Z. Chai, and G. Zhao, “Accelerating methane desorption in lump anthracite modified by electrochemical treatment," International Journal of Coal Geology, vol. 131, pp. 392-399, 2014.

[16] H. Kumar, D. Elsworth, J. Liu, D. Pone, and J. P. Mathews, "Optimizing enhanced coalbed methane recovery for unhindered production and $\mathrm{CO}_{2}$ injectivity," International Journal of Greenhouse Gas Control, vol. 11, pp. 86-97, 2012.

[17] Y. Jiang, X. Song, H. Liu, and Y. Cui, "Laboratory measurements of methane desorption on coal during acoustic stimulation," International Journal of Rock Mechanics and Mining Sciences, vol. 78, pp. 10-18, 2015.

[18] G. Zhang, P. G. Ranjith, B. Wu, M. S. A. Perera, A. Haque, and D. Li, "Synchrotron X-ray tomographic characterization of microstructural evolution in coal due to supercritical $\mathrm{CO}_{2}$ injection at _in-situ_ conditions," Fuel, vol. 255, article 115696, 2019.

[19] G. Zhang, P. G. Ranjith, M. S. A. Perera, Y. Lu, and X. Choi, "Quantitative analysis of micro-structural changes in a bituminous coal after exposure to supercritical CO2 and water," Natural Resources Research, vol. 28, no. 4, pp. 1639-1660, 2019.
[20] H. Hacifazlioglu, "Comparison of efficiencies of microwave and conventional electric ovens in the drying of slime-coal agglomerates," International Journal of Coal Preparation and Utilization, vol. 37, no. 4, pp. 169-178, 2016.

[21] S. Singh, V. B. Neculaes, V. Lissianski et al., "Microwave assisted coal conversion," Fuel, vol. 140, pp. 495-501, 2015.

[22] E. Lester, S. Kingman, and C. Dodds, "Increased coal grindability as a result of microwave pretreatment at economic energy inputs," Fuel, vol. 84, no. 4, pp. 423-427, 2005.

[23] E. Binner, M. Mediero-Munoyerro, T. Huddle et al., "Factors affecting the microwave coking of coals and the implications on microwave cavity design," Fuel Processing Technology, vol. 125, pp. 8-17, 2014.

[24] W. Xia, J. Yang, and C. Liang, "Effect of microwave pretreatment on oxidized coal flotation," Powder Technology, vol. 233, pp. 186-189, 2013.

[25] Z. Peng, J. Y. Hwang, B. G. Kim, J. Mouris, and R. Hutcheon, "Microwave absorption capability of high volatile bituminous coal during pyrolysis," Energy \& Fuels, vol. 26, no. 8, pp. 5146-5151, 2012.

[26] C. Pickles, F. Gao, and S. Kelebek, "Microwave drying of a lowrank sub-bituminous coal," Minerals Engineering, vol. 62, pp. 31-42, 2014.

[27] Y. Wang and N. Djordjevic, "Thermal stress FEM analysis of rock with microwave energy," International Journal of Mineral Processing, vol. 130, pp. 74-81, 2014.

[28] R. Meisels, M. Toifl, P. Hartlieb, F. Kuchar, and T. Antretter, "Microwave propagation and absorption and its thermomechanical consequences in heterogeneous rocks," International Journal of Mineral Processing, vol. 135, pp. 40-51, 2015.

[29] G. Hu, N. Yang, G. Xu, and J. Xu, “Experimental investigation on variation of physical properties of coal particles subjected to microwave irradiation," Journal of Applied Geophysics, vol. 150, pp. 118-125, 2017.

[30] Y. Hong, B. Lin, C. Zhu, and H. Li, "Effect of microwave irradiation on petrophysical characterization of coals," Applied Thermal Engineering, vol. 102, pp. 1109-1125, 2016.

[31] H. Li, L. Tian, B. Huang et al., "Experimental study on coal damage subjected to microwave heating," Rock Mechanics and Rock Engineering, vol. 53, no. 12, pp. 5631-5640, 2020.

[32] H. Li, B. Lin, W. Yang et al., "Experimental study on the petrophysical variation of different rank coals with microwave treatment," International Journal of Coal Geology, vol. 154, pp. 8291, 2016.

[33] J. Huang, G. Hu, G. Xu, N. Yang, J. Xu, and J. Xu, "The development of microstructure of coal by microwave irradiation stimulation," Journal of Natural Gas Science and Engineering, vol. 66, pp. 86-95, 2019.

[34] J. Huang, G. Xu, G. Hu, M. Kizil, and Z. Chen, “A coupled electromagnetic irradiation, heat and mass transfer model for microwave heating and its numerical simulation on coal," Fuel Processing Technology, vol. 177, pp. 237-245, 2018.

[35] R. List, Smithsonian Meteorological Tables, Smithsonian miscellaneous collections, 1951.

[36] A. K. Datta, "Porous media approaches to studying simultaneous heat and mass transfer in food processes. II: property data and representative results," Journal of food engineering, vol. 80, no. 1, pp. 96-110, 2007.

[37] Z. Liu, W. Yang, R. Wu et al., "A new quantitative analysis method for electromagnetic energy dissipation in microwave 
absorption materials," Journal of Magnetism and Magnetic Materials, vol. 516, pp. 167-332, 2020.

[38] J. Wang and T. J. Schmugge, "An empirical model for the complex dielectric permittivity of soils as a function of water content," IEEE Transactions on Geoscience and Remote Sensing, vol. 4, pp. 288-295, 1980.

[39] W. Wagner and A. Pruß, "The IAPWS formulation 1995 for the thermodynamic properties of ordinary water substance for general and scientific use," Journal of Physical and Chemical Reference Data, vol. 31, no. 2, pp. 387-535, 2002.

[40] R. Cherbański and L. Rudniak, "Modelling of microwave heating of water in a monomode applicator - Influence of operating conditions," International Journal of Thermal Sciences, vol. 74, pp. 214-229, 2013.

[41] H. Zhu, T. Gulati, A. K. Datta, and K. M. Huang, "Microwave drying of spheres: coupled electromagnetics-multiphase transport modeling with experimentation. Part I: model development and experimental methodology," Food and Bioproducts Processing, vol. 96, pp. 314-325, 2015.

[42] K. Weltner, "Measurement of specific heat capacity of air," American Journal of Physics, vol. 61, no. 7, pp. 661-662, 1993.

[43] J. M. Forniés-Marquina, J. C. Martín, J. P. Martínez, J. L. Miranda, and C. Romero, "Dielectric characterization of coals," Canadian journal of physics, vol. 81, no. 3, pp. 599610, 2003.

[44] J. Deng, Q. Li, Y. Xiao, C. Shu, and Y. Zhang, "Predictive models for thermal diffusivity and specific heat capacity of coals in Huainan mining area, China," Thermochimica Acta, vol. 656, pp. 101-111, 2017. 